\title{
Social Perception Grading of Hospitals using Fuzzy Knowledge Base Systems
}

\author{
Bhaskar Karn ${ }^{1}$ Praveen Kumar Shukla ${ }^{2}$, Manuj Darbari ${ }^{3}$
}

\begin{abstract}
Proper health is an important parameter to ensure the socioeconomic development of the country. Hospitals are playing vital role to improve the health standards in the life and serves the society very effectively. The assessment of quality and ease of medical facilities provided by the hospitals is an important research line. The higher quality of medical care improves the patient satisfaction leading to social perception enhancement. In this paper, we are investigating a new Expert System to assess the quality of medical care of any hospital depending on few parameters. The system is developed using fuzzy knowledge based systems and implemented in Guaje. The system would be generating the grades of different hospitals as per the quality care provided by them.
\end{abstract}

Keywords: Fuzzy Logic, Fuzzy Knowledge Base System, Interpretability, Social Perception.

\section{Introduction}

Health is an important parameter for the economic and social growth of any country. The increasing pollutions and mixing in food items

resulted into big problem of deteriorating health in the society [1]. At result we get exponential growth in the number and types of chronic health problems. Due to exceptional growth in the diseases, the demand of hospital and medical services has incremented in the society. As per the definition given by WHO (World Health Organization), the hospital is an integral part of social and medical organization, the function of which, is to provide complete health care for the population. The health care may be preventive and curative. Apart from this, the hospitals also provide the training to health workers and carry out biosocial research. The government has opened many hospitals in the cities and even in rural areas. The private hospitals and paramedical institutes are also playing vital role in the sector social health care.

The public preview about the quality of medical care delivered by any hospital is the social perception of the hospitals. Due to growth of number of hospitals, it is important to quantify the social perception of hospitals that can be used by the public to get better medical care. Several parameters are identified to assess the social perception of the hospitals. These identified parameters are as follows; behavior of medical staff, availability of emergency medical services, availability of medical instruments, behavior of doctors, ease of distance, cost of medical facilities, cleanliness in the hospitals etc.

We have proposed a Knowledge Base System (KBS) which estimates and calculates the Social Perception Index (SPI) for all the hospitals depending on the parameters discussed above. This KBS is developed using Fuzzy Logic and implemented in the open access software 'Guaje' $[17,18]$. The interpretability and accuracy parameters are also identified and evaluated during the experiment. 


\section{Related work}

Fuzzy logic [2,3] and set theory is the expanded version of Boolean and crisp logic and set theory. It deals with the imprecision, uncertainty, partial truth and vagueness of the information of any system. It is basically the implementation of the human decision making and handles the linguistic information based systems. These are qualitative, descriptive and subjective which may contain overlapping degrees and represented by the membership functions. The membership functions produce membership grade $\mu$ with the condition $0 \leq \mu \leq 1$ for each possible value of fuzzy predicate $[4,5]$.

Several issues are concerned during the development of fuzzy knowledge based systems. The interpretability and accuracy parameters assess the performance and efficiency of the proposed system.

Interpretability [15] is the quality of designed system which shows if its behaviour is human understandable or not by seeing its functioning. On the other hand, accuracy is the feature in which the closeness between real and designed system is judged. Accuracy and interpretability features are contradictory with each other; one can be improved at the cost of the other. This is called the interpretability-accuracy trade-off $[6,8,14]$. Few of the knowledge base systems are also developed in advanced fuzzy methods, like interval type-2 fuzzy sets $[7,13]$.

Evolutionary multi-objective optimization is one of the strategies to deal with interpretabilityaccuracy trade-off fuzzy knowledge base system or fuzzy classifiers [9-12].

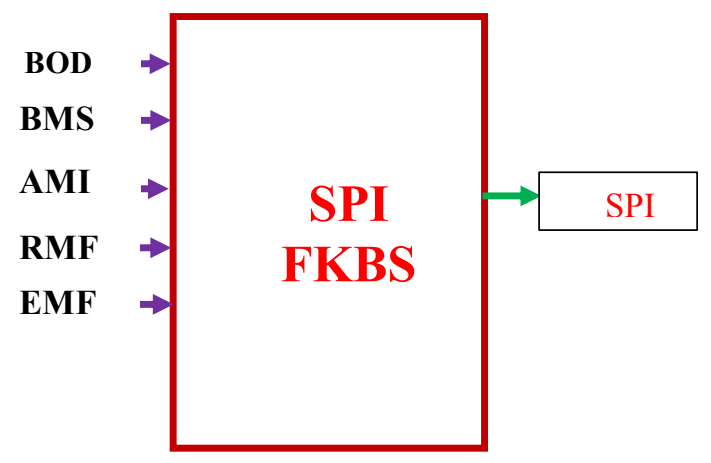

Fig. 1. Block diagram for SPI-FKBS

The parameters of the system are explained in Table 1 given below.

Table 1. Input parameters of SPI-FKBS

\section{Proposed System for grading of Social Perception}

The working of SPI-KBS is based on five input parameters; behavior of doctor (BOD), behavior of medical staff (BMS), availability of medical instruments (AMI), rate of medical facilities (RMF), availability of emergency medical facility (EMF).

\section{Implementation and results}

The proposed system has been implemented using open access software tool "Guaje" developed by Alonso and Magdalena [17, 18]. The rule base generation methods are Fuzzy Decision Tree Method and Wang Mendel Method [19]. 
Proc. of the Fifth Intl. Conf. Advances in Computing, Communication and Information Technology- CCIT 2017 Copyright (C) Institute of Research Engineers and Doctors, USA .All rights reserved. ISBN: 978-1-63248-131-3 doi: 10.15224/ 978-1-63248-131-3-16

The data for this experiment has been collected from the patients of different hospitals at Lucknow, UP, India. The description of data set is as follows;

Table 2. Characteristics of data set

\begin{tabular}{cll}
\hline S. No. & Characteristics & Value \\
\hline 1 & Type & Classification \\
2 & Number of attributes & 5 \\
3 & Number of instances & 250 \\
4 & $\begin{array}{l}\text { Attribute } \\
\text { characteristics }\end{array}$ & Integer \\
\hline
\end{tabular}

In the experiment the membership functions of attributes and their data distribution are as follows;

\section{Attribute 1: BOD}

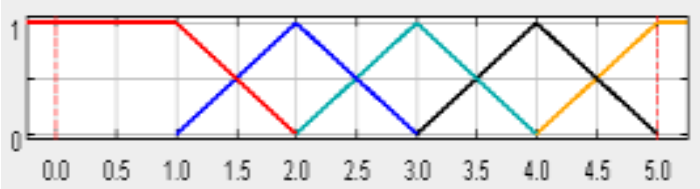

Fig. 2. Membership function of BOD

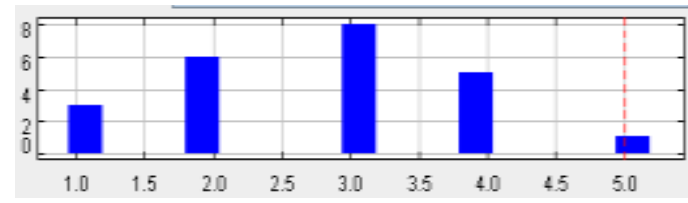

Fig. 3. Data distribution of BOD

\section{Attribute 2: BMS}

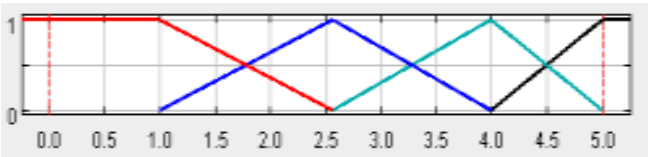

Fig. 4. Membership function of BMS

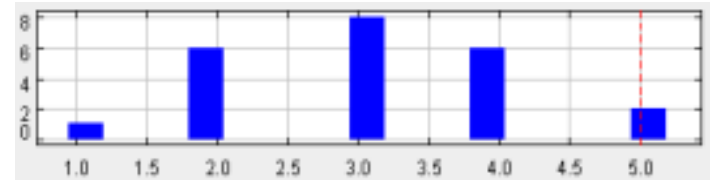

Fig. 5. Data distribution of BMS

\section{Attribute 3: AMI}

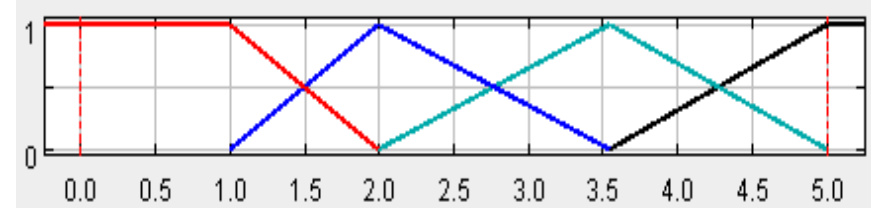

Fig. 6. Membership function of AMI

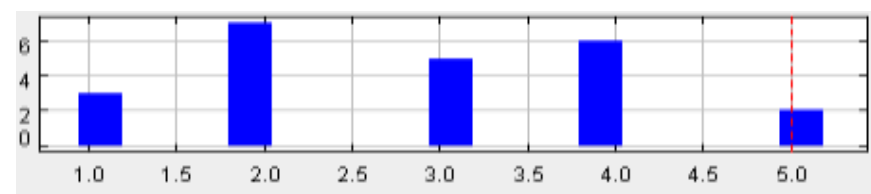

Fig. 7. Data distribution of AMI

\section{Attribute 4: RMF}

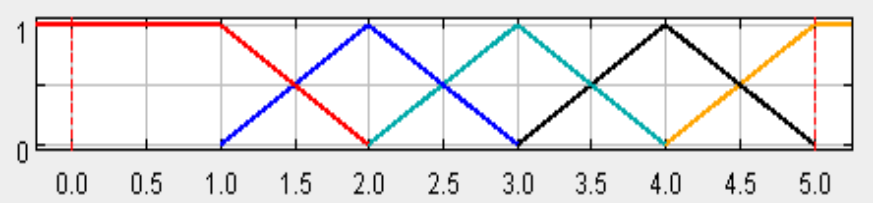

Fig. 8. Membership function of RMF

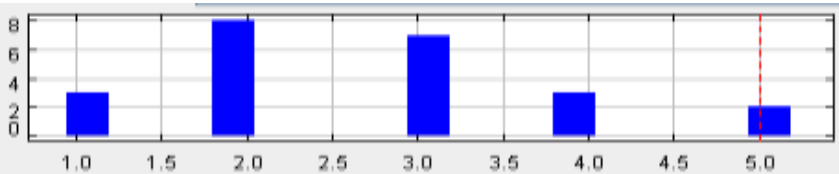

Fig. 9. Data distribution of RMF

\section{Attribute 5: EMF}

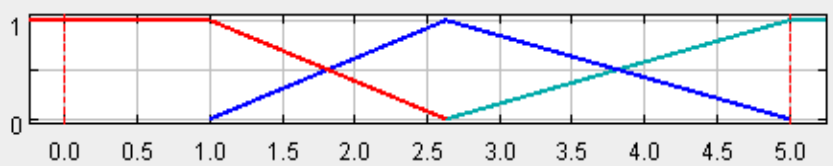

Fig. 10. Membership function of EMF

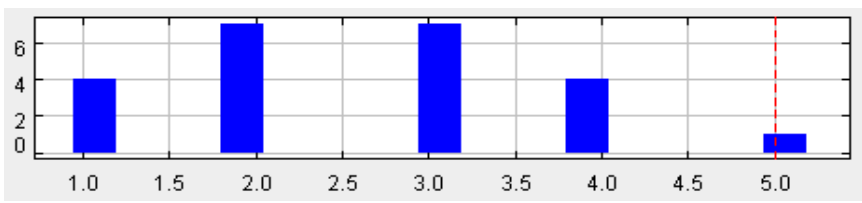

Fig. 11. Data distribution of EMF 


\section{Attribute 6 SPI FKBS}

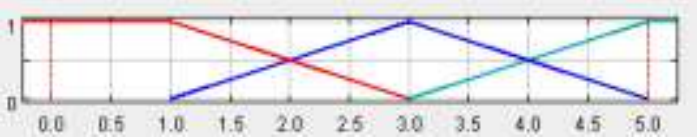

Fig. 12. Membership function of SPI-FKBS

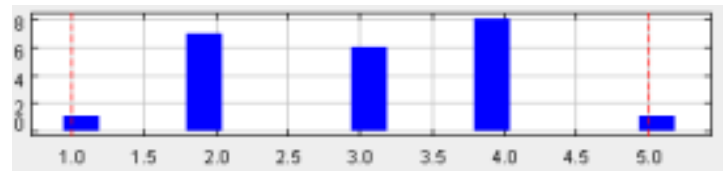

Fig. 13. Data distribution of SPI-FKBS

\subsection{Experimental results}

\subsubsection{Fuzzy decision trees}

The settings of the experiment are as follows, Maximum tree depth $=5$

Minimum significant level $=0.2$

Leaf minimum cardinality $=1$

Tolerance threshold $=0.1$

Relative loss of performance $=0.1$

Coverage threshold $=0.9$

Minimum entropy $=0.001$

Table 3. Experimental results in Fuzzy Decision Tree Method

\begin{tabular}{lccc}
\hline \multicolumn{1}{c}{ Interpretability } & \multicolumn{2}{c}{ Error } \\
\hline $\begin{array}{l}\text { Nauck's } \\
\text { Index } \\
\text { NOR }\end{array}$ & 0.008 & MSE & 0.834 \\
TRL & 31 & SE & 1.026 \\
ARL & 122 & & \\
ARC & 3.935 & & \\
TFR & 30.036 & & \\
IFR & 12.032 & & \\
LVI & 2.348 & \\
\hline
\end{tabular}

\subsubsection{Wang Mendel Method}

\begin{tabular}{lclc}
\hline \multicolumn{2}{c}{ Interpretability } & \multicolumn{2}{c}{ Error } \\
\hline Nauck's & 0.01 & MSE & 0.984 \\
Index & 21 & RMSE & 1.403 \\
NOR & 105 & & \\
TRL & 5 & & \\
ARL & 21.036 & & \\
ARC & 6 & & \\
TFR & 1.348 & & \\
IFR & 0.478 & & \\
LVI & & \\
\hline
\end{tabular}

Table 4. Experimental results in Wang Mendel Method

$\mathrm{ARC}=$ Average Rule Complexity, $\mathrm{NOR}=$ Number of Rules, $T R L=$ Total Rule Length, $A R L=$ Average Rule Length, TFR=Theoretical Fired Rules, IFR=Inferential Fired Rules, LVI=Logical View Index.

\section{Conclusion}

Fuzzy based knowledge base systems have proven their capabilities in the implementation of human like decision making. In this paper, we have proposed and implemented the fuzzy knowledge based system for making decision about the social perception of hospitals. The system has been developed based on the data collected by patients from the different hospitals of the Lucknow, UP, India. The developed system is found interpretable and accurate as per the results of various experiments carried out.

\section{References}

1. Tiwari, K., Singh, P., Kumar, S.: A Review on Effective Role Performance of Private Medical Institutes towards Society. Journal of Business Management 3(12) 2015 25-30

2. Zadeh, L. A.: Fuzzy sets, Information \& Control, 8 (3) (1965) 338-353

3. Zadeh, L. A., A fuzzy set theoretic interpretation of linguistic hedges. Journal of Cybernetics 2 (3) (1972) 4-34

4. Zadeh, L. A., The concept of linguistic variable and its application to approximate reasoning -I, Information Sciences, 8(3) (1975) 199-249

5. Zadeh, L. A.: Fuzzy sets as a basis of possibility, Fuzzy Sets Systems, 1 (1) (1978) 3-28 1978

6. Shukla, P. K., Tripathi, S. P.: A review on the interpretability-accuracy trade-off in evolutionary multi-objective fuzzy systems (EMOFS). Information 3(3) (2012) 256-277 
7. Shukla, P. K., Tripathi, S. P.: A new approach for tuning interval type-2 fuzzy knowledge bases using genetic algorithms. Journal of Uncertainty Analysis and Applications 2 (2014) 1-15

8. Shukla, P. K., Tripathi, S. P.: Handling high dimensionality and interpretability-accuracy tradeoff issues in evolutionary multi-objective fuzzy classifiers, International Journal of Scientific \& Engineering Research 5(6) (2014)1-6

9. Shukla, P. K., Tripathi, S. P.: Interpretability and accuracy issues in evolutionary multi-objective fuzzy classifiers, International Journal of Soft Computing and Networking 1(1) (2016) 55-68

10. Shukla, P. K., Tripathi, S. P.: A Survey on Interpretability-Accuracy Trade-Off in Evolutionary Fuzzy Systems, IEEE International Conference on Genetic and Evolutionary Computation (ICGEC 2011), Japan, 29 August-01 September, 2011

11. Shukla, P. K., Tripathi, S. P.: On the Design of Interpretable Evolutionary Fuzzy Systems (I-EFS) with Improved Accuracy, International Conference on Computing Sciences, LP University, India, Sept. 2012

12. Shukla, P. K., Tripathi, S. P.: Interpretability Issues in Evolutionary Multi Objective Fuzzy Knowledge Base Systems, 7th International Conference on BioInspired Computing: Theories and Applications (BIC-TA 2012), ABVIIITM, Gwalior, India, 14-16 December, 2012

13. Sanz, J. A., Fernandez, A., Bustince, H.: IVTURS: A linguistic fuzzy rule based classification system based on a new interval valued fuzzy reasoning method with tuning and rule selection. IEEE Transactions on Fuzzy Systems 21(3) (2011) 399411

14. G.-Hernández, M., Sainz-Palmero, G. I. and FuenteAparicio, M. J.: Complexity reduction and interpretability improvement for fuzzy rule systems based on simple interpretability measures and indices by bi-objective evolutionary rule selection. Soft Computing 16(3) 2012 451-470

15. Gacto, M. J., Alcala, R., Herrera, F.: Interpretability of linguistic fuzzy rule based systems:

16. an overview on interpretability measures, Information Sciences. 181(20) (2011) 4340-4360

17. Alonso, JM, Magdalena, L: HILK++: an interpretability guided fuzzy modeling methodology for learning readable and comprehensible fuzzy rule based classifiers. Soft. Comput. 15(10) (2011) 1959-1980

18. Alonso, JM, Magdalena, L: Generating understandable and accurate fuzzy rule based systems in a Java environment. In: Fanelli, AM, Pedrycz, W, Petrosino, A (eds.) Fuzzy Logic and Applications, 9th International Workshop, WILF 2011, Trani, Italy, August 29-31, 2011. Lecture Notes in Artificial Intelligence, vol. 6857, pp. 212219. Springer, Berlin (2011)

19. Wang, L. X., Mendel, J. M., Generating fuzzy rule by learning from examples, IEEE Transactions on Systems, Man and Cybernetics. 22(6) 1414-1427 (1992)
About Author (s):

1

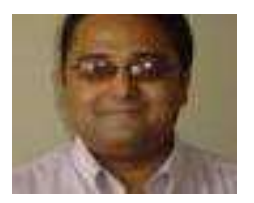

Department of Computer Science and Engineering, B I T Mesra, Ranchi.

(Corresponding Author)

2

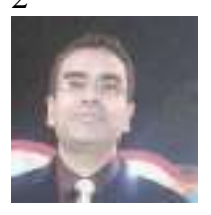

Department of Information Technology, Babu Banarasi Das Northern India Institute of Technology, Lucknow, India

3

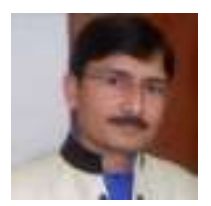

Department of Information Technology, Babu Banarasi Das National Institute of Technology \& Management, Lucknow, India 